\title{
Improved In Vivo Subcutaneous Tumor Generation by Cancer Cell Sheet Transplantation
}

\author{
JUN AKIMOTO, MASAMICHI NAKAYAMA, SOICHI TAKAGI and TERUO OKANO \\ Institute of Advanced Biomedical Engineering and Science, \\ Tokyo Women's Medical University (TWIns), Tokyo, Japan
}

\begin{abstract}
Background/Aim: In vivo subcutaneous tumor models are generally prepared by the injection of a cancer cell suspension to evaluate the pharmaceutical effects on tumor tissues. However, dispersed cells show low biological activities because of enzyme-induced cell harvest treatment, thus limiting the formation of tumor tissues. In this study, a biologically active cancer cell monolayer (cell sheet) was used to improve the efficiency of subcutaneous tumor formation. Materials and Methods: Mouse lung squamous cancer cells (KLN-205) were transplanted on the subcutis of immunocompetent and immunodeficient mice in the form of a dispersed cell suspension or cell sheet, and the tumor formation abilities were independently investigated with considering immunological effects. Results: Mouse lung squamous cancer cells (KLN-205) scarcely formed malignant tumors on the mouse subcutis following injection of the cell suspension. On the other hand, cell transplantation in the cell sheet form successfully achieved effective tumor development due to only weak immunological reactions at the transplanted area. And thus, the cancer cells maintained their proliferative activity to form tumors. Conclusion: Transplantation of the cell sheet is effective to generate subcutaneous tumor-bearing mice, providing a useful alternative to the low tumor formation activities induced with the conventional injection method.
\end{abstract}

In preclinical tests of new anti-cancer drugs, subcutaneous tumor-bearing animal models are commonly used to evaluate

This article is freely accessible online.

Correspondence to: Masamichi Nakayama and Teruo Okano, Institute of Advanced Biomedical Engineering and Science, Tokyo Women's Medical University, 8-1 Kawada-cho, Shinjuku-ku, Tokyo, 162-8666, Japan. Tel: +81 333538112 (Ext. 43215), e-mail: nakayama.masamichi@twmu.ac.jp, tokano@twmu.ac.jp

Key Words: Cell sheet, tumor-bearing mice, transplantation, cancer cell, immunological reaction. the in vivo anti-tumor activities of drug candidates. Currently, huge numbers of cancer cell lines are supplied from official cell banks or suppliers to prepare tumor-bearing animals. In addition, the use of primary cancer cells from patients would promote the development of personalized cancer chemotherapy $(1,2)$. However, the cells transplanted on the subcutis of animals show limited ability to develop tumor tissues. The most fundamental problem in preparing tumor-bearing animals is the low graft survival rate of cells transplanted to host tissues. Cultured adhering cancer cells are commonly harvested from a cell culture dish by enzyme digestions, and the dispersed cells are injected directly to induce the formation of in vivo tumors. However, the subcutaneous injection method results in a low cell engraftment efficiency and it is difficult to control the size of the tumors because the enzyme treatment causes the cells to lose their extracellular matrix (ECM) and cell-cell junctions. To overcome this issue, the cancer cell transplantation method should be improved. Our laboratory has successfully developed a technology for generating a highly bioactive cell monolayer (i.e., a cell sheet) by using a temperature-responsive culture dish without requiring enzymatic digestion (3). The cell sheet prepared in this manner could maintain the biologically intact structure of cell-cell junctions and preserve the basal ECM proteins, allowing for the cells to immediately and tightly adhere on the living tissues solely by patching on target areas (4). Our previous studies showed that the transplantation of a cell sheet could increase the engraftment efficiency by more than 10 times compared with that achieved with cell suspension injection $(5,6)$. Furthermore, using this technique to prepare tumor-bearing animals by transplanting cancer cell sheets, tumor formation at the subcutis or orthotopic organs (e.g., the ovary) of mice was successfully improved $(6,7)$. In this study, we investigated the difference in tumor formation activity following transplantation of mouse lung squamous cancer cells (KLN-205) as a cell suspension and as a cell sheet to confirm the influence of the immune reaction on engraftment ability on immunocompetent (IC) and immunodeficient (ID) mice. Using these two models, the tumor formation mechanism of the cancer cell sheet was explored based on histological analysis of the tumor specimens at the early stage after transplantation. 


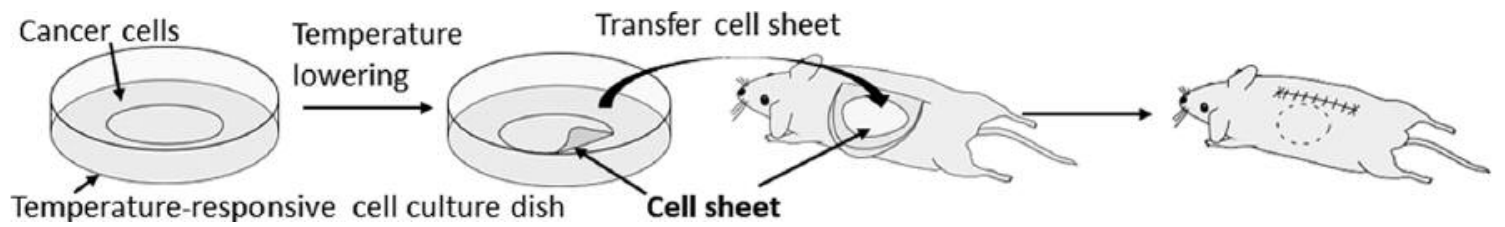

Figure 1. Procedure of KLN-205 cell sheet transplantation on the mouse subcutis.

\section{Materials and Methods}

Cell culture. KLN-205 cells (Dainippon Sumitomo Pharma, Osaka, Japan) were cultured in Eagle's minimum essential medium (SigmaAldrich, St. Louis, MO, USA) supplemented with $1 \%$ non-essential amino acids (Sigma-Aldrich, St. Louis, MO, USA), 10\% fetal bovine serum (Japan Bioserum, Hiroshima, Japan), and antibiotics (Gibco, Grand Island, NY, USA). The luciferase gene was transfected into KLN-205 cells using Ready-To-Use Lentivirus Marker Supernatant: Lenti-Luciferase (Biogenova, Rockville, MD, USA) according to a standard protocol supplied by the manufacturer.

Animals and animal care. Thirteen-week-old female BDF1 mice (Japan SLC, Hamamatsu, Japan), as the IC group, and BALB/c $n u / n u$ mice (Japan SLC), as the ID group, were maintained in a barrier facility on high-efficiency particulate air-filtered racks with 12-h dark/light cycles. Animal experiments were performed in accordance with the experimental procedure approved by the Committee for Animal Research of Tokyo Women's Medical University.

Transplantation of cancer cells. Cancer cell sheet was prepared and subcutaneously transplanted in the mice according to the previous report (Figure 1) (6). Briefly, cancer cells were cultured on a temperature-responsive cell culture dish (UpCell ${ }^{\circledR}$, CellSeed, Tokyo, Japan) with a $15-\mathrm{mm}$ circular culture area at $37^{\circ} \mathrm{C}$ for 4 days. A cell sheet $\left(5.0 \times 10^{5}\right.$ cells $)$ was harvested from $\mathrm{UpCell}^{\circledR}$ after reducing the incubation temperature to $20^{\circ} \mathrm{C}$, and was transplanted on the mouse subcutis. Cell transplantation was also performed with injection of a cancer cell suspension $\left(100 \mu 1,5.0 \times 10^{5}\right.$ cells $)$. In addition, the negative control mice were prepared using subcutaneous injection of physiological saline $(100 \mu \mathrm{l})$. Tumor volume was measured using a vernier caliper and approximated as a semi-ellipsoid. Bioluminescence emitted from KLN-205 cells was measured by an in vivo imaging system (IVIS ${ }^{\circledR}$ Lumina, PerkinElmer, Waltham, MA, USA). All images were taken at $12 \mathrm{~min}$ after the intraperitoneal injection of beetle luciferin $(300 \mathrm{mg} / \mathrm{kg}$; Promega, Madison, WI, USA).

Histological analysis of cell sheets and tumor tissues. Mouse subcutaneous tissues around the KLN-205 cell-transplanted area were fixed with $4 \%$ paraformaldehyde (Muto Pure Chemical, Tokyo, Japan) and embedded in paraffin. The specimens were stained with hematoxylin and eosin (HE) and Azan. Immunostainings of cytokeratin-5 (CK5) and Ki-67 were performed as follows. Preparations were treated with target retrieval solution (Agilent, Santa Clara, CA, USA), and endogenous peroxidase was blocked with Dako REAL peroxidase-blocking solution (Agilent, Santa Clara, CA, USA). The blocking process was performed using Blocking One Histo (Nacalai Tesque, Kyoto, Japan). The section was treated with anti-CK5 (ab53121, Abcam, Cambridge, UK) or anti-Ki67 (ab66155, Abcam, Cambridge, UK), followed by incubation with horseradish peroxide-conjugated goat anti-mouse IgG H\&L (ab97023, Abcam, Cambridge, UK). The sections were dyed with 3,3-diaminobenzidine, and the nuclei were stained with hematoxylin.

\section{Results}

Tumor growth. Luciferase gene-transfected KLN-205 cells were transplanted on the subcutis of IC and ID mice in the form of a dispersed cell suspension or cell sheet. Figure 2 shows the volume changes of tumors and the gross appearance of developed tissues on the subcutis. All the cell -injected mice formed tumors on the transplanted area within 5 days after transplantation. However, in 6 ID mice $(n=12)$ and 5 IC mice $(n=8)$, the tumors disappeared at 14 days posttransplantation, and tumor growth of the other mice was negligible. In contrast, all of the cell sheet-transplanted mice ( $n=11$ for ID and $n=8$ for IC) developed tumors on the subcutaneous tissue, and the volume of the tumor gradually increased, except for one ID and one IC mouse. Subcutaneous tumor tissues developed at 5-7 days and 7-14 days posttransplantation in the ID and IC mice, respectively. The mean volume of the subcutaneous tumors in the ID and IC mice at day 14 was $142 \pm 108 \mathrm{~mm}^{3}$ and $82.1 \pm 34.0 \mathrm{~mm}^{3}$, respectively. Tumor growth was also determined by the luciferin emission from KLN-205 cells. Figure 3 shows the bioluminescent intensity changes of KLN-205 cell-transplanted mice and bioluminescent images captured around the cell-transplanted area. In the cell suspension-transplanted mice, the luciferinderived bioluminescence from KLN-205 cells was detected at the cell-transplanted area at 1 day post-transplantation. However, both the ID and IC mice rapidly decreased their bioluminescent intensities, and then the bioluminescence completely disappeared around the transplanted area in all mice except for one. In contrast, the bioluminescence intensities increased in all of the cell sheet-transplanted ID mice after the transplantation. Of note, the bioluminescent intensities initially decreased at the early stage in IC mice, 

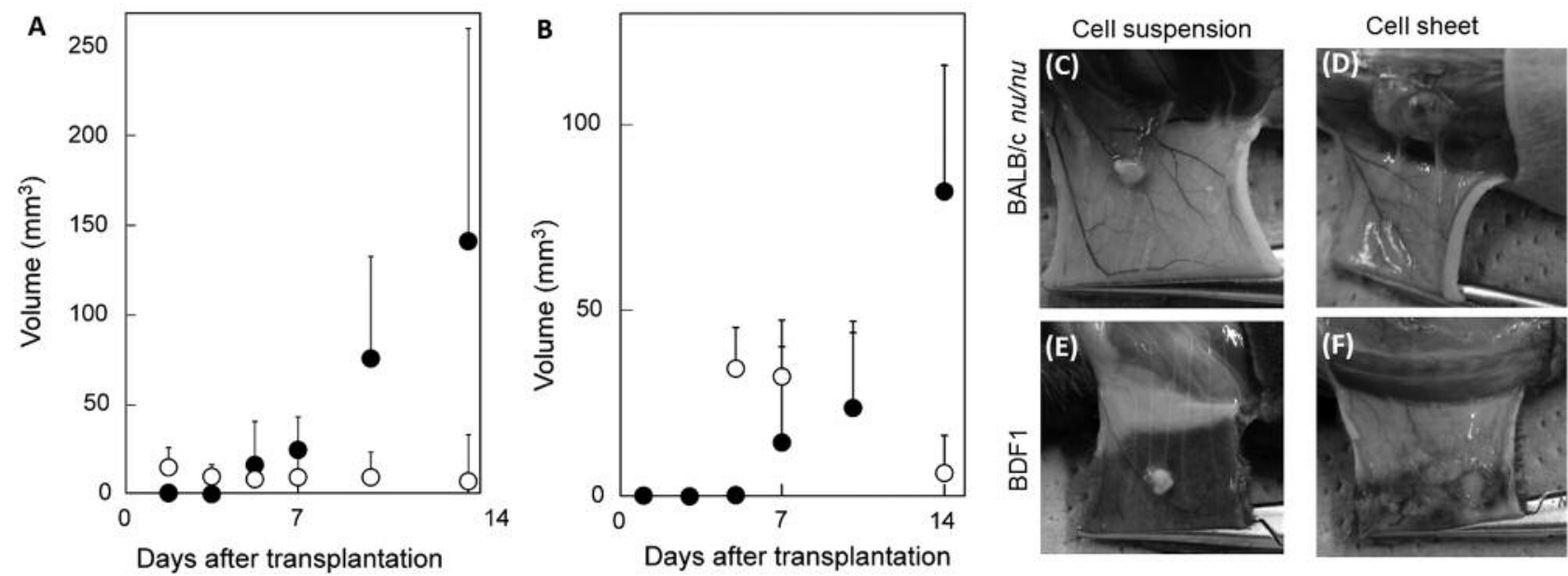

Figure 2. Growth curves and gross appearance of developed tumors. $(A, B)$ Volume changes of subcutaneous tumors developed on (A) BALB/c nu/nu and (B) BDF1 mice. The open and closed circles represent the volumes of the tumors generated by the transplantation of a cell suspension and cell sheet, respectively. $(C-F)$ Representative images of tumors generated on the subcutaneous tissue of BALB/c nu/nu mice by $(C)$ cell suspension injection and $(D)$ cell sheet transplantation, and of BDF1 mice by $(E)$ cell suspension injection and $(F)$ cell sheet transplantation at 14 days after transplantation.
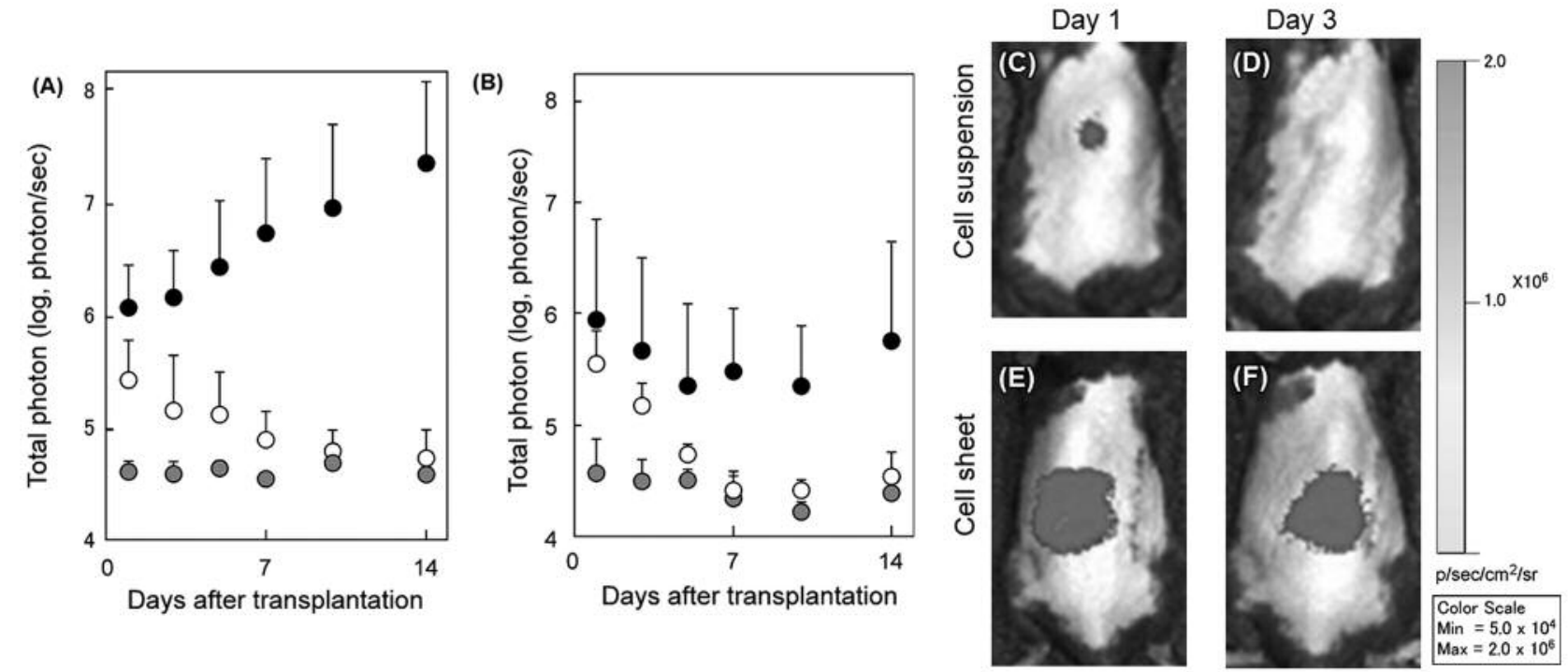

Figure 3. Bioluminescent intensity from luciferin-transfected $K L N-205$ cells on (A) BALB/c nu/nu and (B) BDF1 mice. Open circles: cell suspension; closed circles: cell sheet; and gray circles: saline (negative control). Representative bioluminescent images generated from luciferin-transfected $K L N-205$ cells transplanted on BDF1 mice using $(C, D)$ a cell suspension and $(E, F)$ cell sheet (left column: day 1, right column: day 3$)$.

and were maintained and then slightly increased along with tumor growth from 5-7 days post-transplantation.

Histological analysis. Tumor tissues were sampled from the mouse subcutaneous tissues at 14 days post-transplantation. Figure 4 shows the images of HE staining, Azan staining, and CK5 immunostaining of the KLN-205 cell sheet and tumors formed in IC mice. The HE staining image showed that the cell suspension-induced tumors contained numerous inflammatory cells with no KLN-205 cells in both IC and ID mice. In addition, staining of a squamous cancer cell marker, CK5 (8), was performed to identify the KLN-205 cells in 

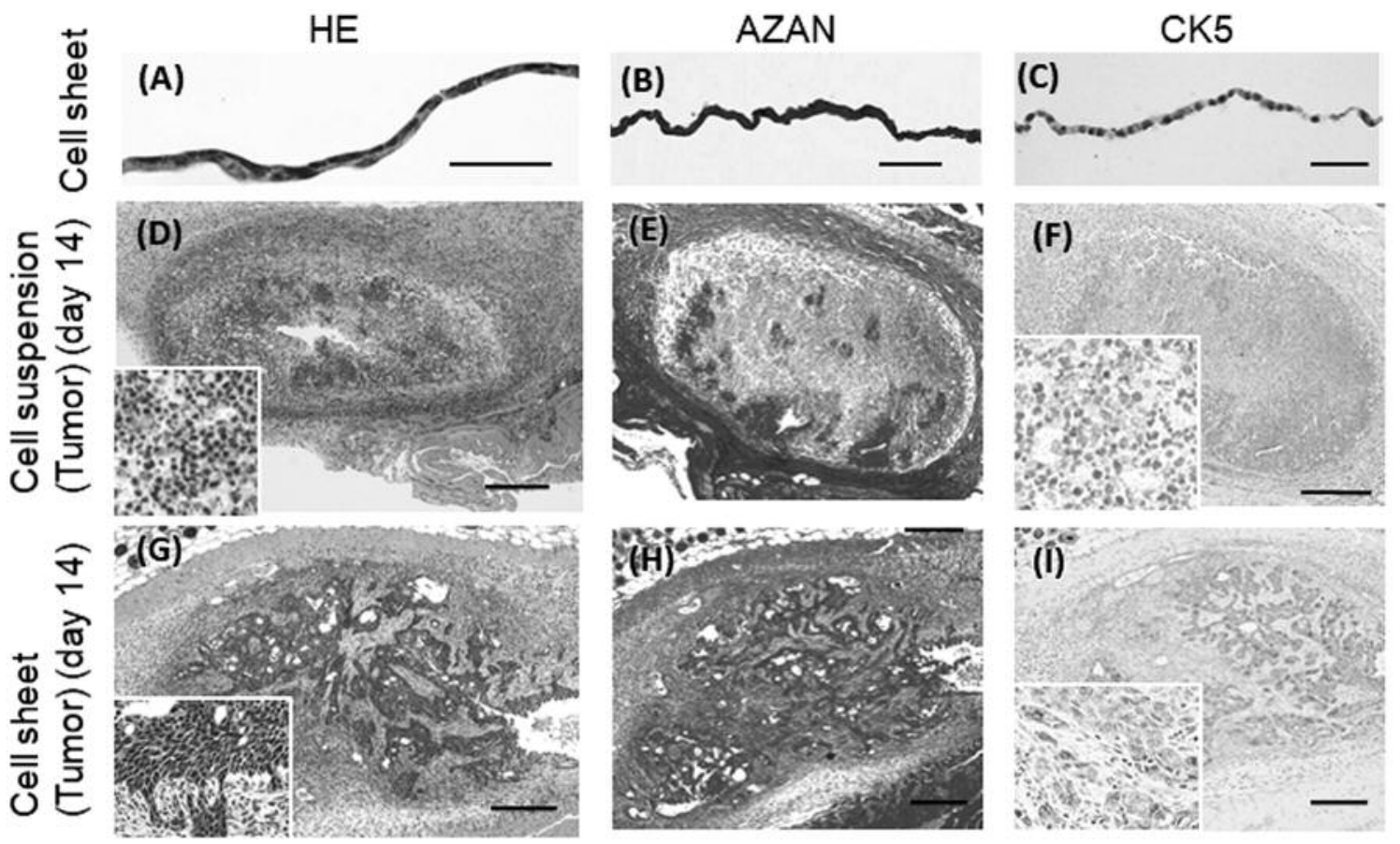

Figure 4. Cross sections of $(A-C)$ the $K L N-205$ cell sheet, and subcutaneous tumors developed from $(D-F)$ the cell suspension and (G-I) the cell sheet on BDF1 mice at 14 days post-transplantation. ( $A, D, G)$ Hematoxylin and eosin (HE) staining, $(B, E, H)$ Azan staining, and $(C, F, I)$ immunostaining of $C K-5$. The insets at the lower left corner of each image are magnified images. Scale bars: (A-C) $50 \mu m,(D-I) 250 \mu m$.

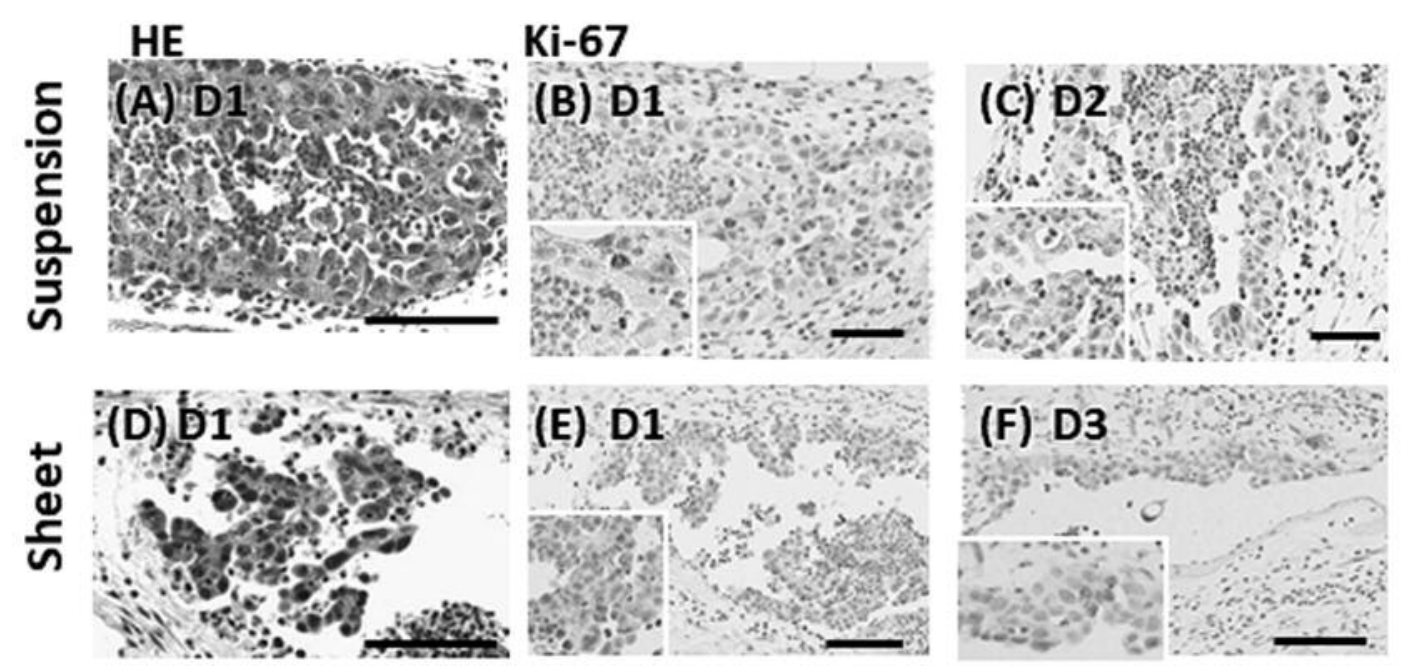

Figure 5. Cross-sections of BDF1-derived subcutaneous tissues adjacent to the KLN-205 cells-transplanted area. Hematoxylin and eosin (HE) staining and immunostaining images of Ki-67 of subcutaneous tissues. (A-C) Cell suspension- and (D-F) cell sheet-transplanted tissues. The insets at the lower left corner of each image are magnified images. Scale bars: $100 \mu \mathrm{m}$.

tumor tissues. Although KLN-205 cells strongly expressed CK5 in the cell sheet before transplantation, no CK5-positive cells were confirmed on the tumor tissues developed from the dispersed KLN-205 cells. Therefore, the cell suspension- derived tumors were identified as granulation tissues. In contrast, the KLN-205 cell sheet-derived tumors exhibited a mixture of weak and strong eosinophilic cells in the HEstained image. In the strong eosinophilic domain, the cells 
formed an epithelial structure and strongly expressed CK5 In addition, these cells possessed atypical nuclei, compared with other normal cells. According to these findings, the eosinophilic cells were identified as KLN-205 cells. Moreover, the cell sheet-induced tumors consisted of numerous CK5-negative cells. These cells were confirmed as fibroblastic cells because of the clear blue color resulting from Azan staining. Comparable histological features were observed in the subcutaneous tumor that developed in one of ID the mice that received the cell sheet transplantation (data not shown).

Characterization of transplanted cancer cells in the initial period. Transplanted KLN-205 cells were analyzed at the early period post-transplantation using the subcutaneous tissue specimens collected adjacent to the cell transplantation area in IC mice (Figure 5). The transplanted KLN-205 cells were found in one or two tissue sections each day $(n=3)$. In addition, KLN-205 cells were not found in the cell-injected mice on day 3 or later. At 1 day posttransplantation, immunological cells, including neutrophils, were mainly found in the transplanted area, regardless of the transplantation method. In particular, numerous neutrophils were closely localized around KLN-205 cells at the cellinjected area. Injected KLN-205 cells retained the neutrophils around the transplanted area, whereas the number of neutrophils was clearly reduced from the cell sheet method. In this study, the immunological reaction induced by the saline injection was also investigated as the negative control, and a negligible reaction was observed (data not shown).

In the next step, to investigate the proliferative activity of transplanted KLN-205 cells, the expression of Ki67 was estimated, as a cell proliferation marker (9). Although Ki67 expression was observed before transplantation, the Ki67 expression level of transplanted KLN-205 cells decreased in the subcutaneous tissue on day 1. A portion of the KLN-205 cells showed increased Ki67 expression at 3 days posttransplantation, and then the expression further increased substantially at day 14 . In contrast, no Ki67 expression was observed in the KLN-205 cells transplanted by the injection method at day 3 and later.

\section{Discussion}

A cancer cell sheet was transplanted on the subcutaneous tissue of ID and IC mice to investigate its tumor formation ability with consideration of immunological effects. Our previous study showed that the cell sheet transplantation method could improve engraftment efficiency and subsequent tumor growth of mouse breast cancer $4 \mathrm{~T} 1$ cells in IC mice, compared with the conventional method of injection of a cancer cell suspension (6). In this study, histological analysis of cancer cell-transplanted tissues was performed to investigate the tumor growth mechanism of cell sheet-formed cancer cells. Injection of KLN-205 cells resulted in severe subcutaneous engraftment to the host tissues, as shown in Figure 4. The transplanted cells were immediately excluded from the transplanted area, and a strong inflammation reaction was continuously observed. In addition, the bioluminescence analysis showed that the IC mice more rapidly excluded the transplanted KLN-205 cells from the body compared with the ID mice. This promotion in rejection of the transplant indicated that cell elimination was most likely attributed to an immunological effect. Therefore, minimizing the immunological response caused by cell transplantation is considered to be a significant factor for improving the engraftment of cancer cells on host tissues in fabricating tumor-bearing mice. In this study, the cell sheet transplantation method improved the early survival rate of transplanted cells on the subcutis. The bioluminescent study showed that the cell sheet method preserved cells on the host subcutis, compared with the cell-injection method. This result is attributed to the fact that the cell sheet maintained a biologically intact structure, and thus adhered on the host tissue surface without any transplantation loss. Considering that the procedure of cell transplantation involves skin exfoliation and suture, the cell sheet method seems to be more invasive than the simple injection method. In fact, a significant immunological reaction was observed around the transplanted area at 1 day after cell sheet transplantation (Figure 5B). In addition, the proliferation activity of transplanted cancer cells decreased immediately after transplantation. These results indicated that the immunological reaction at the early stage decreased the graft survival of transplanted cells. However, the number of immunological cells was negligible at 3 days post-cell sheet transplantation, and the baseline cell proliferation activity was retrieved within 3 days post-cell sheet transplantation. Actually, the growth behavior corresponded to the results of changes in luciferin-derived bioluminescence and tumor volumes. By contrast, the injection method of dispersed cells induced a strong inflammation reaction and continuously retained immunological cells, mainly around floating cells inside the cell-injected tissue. The histological analysis showed that non-adhering cells were attacked by immunological cells and showed scarce proliferative activity after transplantation. Therefore, non-adhering cells were one of the factors contributing to the activation of the inflammation reaction, and thus the inflammatory cells probably suppressed the activity of the transplanted cells. The cancer cell suspension recovered from culture dishes lost the cell-cell junctions and ECM proteins. Thus, the cell injection method resulted in the introduction of numerous 
cells with low adhesive properties into host animals. Because an inflammation reaction was scarcely observed following saline injection (data not shown), non-adhering cells appear to be the most likely inflammation-inducing factors. Consequently, dispersed cells were unable to form cancer tissue on the mouse subcutis due to the strong and successive inflammation reaction induced. From these results, the possible growth process of transplanted cancer cells in the sheet form is proposed as follows. 1) At the early stage, most of the transplanted cancer cells in the sheet form showed low proliferation activities. Owing to the high survival rate of transplanted cancer cells in the recipient body, cell sheet-formed cancer cells avoided exposure to a strong immune response, compared with dispersed cells. 2) Next, the inflammation reaction almost completely disappeared, and the cancer cells gradually retrieved their proliferation activity (3). Finally, cancer cells proliferated inside the subcutis and achieved cancer tissue formation.

A KLN-205 cell sheet engrafted on the mouse subcutis showed rapid tissue adhesiveness. The adhesive property of the cell sheet resulted in high host engraftment efficiency with a low immune response, compared with the dispersed cancer cells transplanted following enzymatic treatment. Consequently, transplantation in the cell sheet form allowed for the generation of malignant tumors on the subcutis, even in IC mice. The results of this study demonstrate that cell sheet transplantation is a promising method for the establishment of subcutaneous tumor-bearing animal models for drug screening and cancer-related research.

\section{Acknowledgements}

This research was supported by JSPS KAKENHI Grant Number 25282145 and A3 Foresight Program "Nano-Biomaterials and Delivery Strategies in Regenerative Medicine for Intractable Diseases" from Japan Society for the Promotion of Science (JSPS).

\section{References}

1 Reddel RR, Murphy LC, Hall RE and Sutherland RL: Differential sensitivity of human breast cancer cell lines to the growth-inhibitory effects of tamoxifen. Cancer Res 45(4): 1525-1531, 1985.

2 Bishop PC, Myers T, Robey R, Fry DW, Liu ET, Blagosklonny MV and Bates SE: Differential sensitivity of cancer cells to inhibitors of the epidermal growth factor receptor family. Oncogene 21(1): 119-127, 2002.

3 Okano T, Yamada N, Sakai H and Sakurai Y: A novel recovery system for cultured cells using plasma-treated polystyrene dishes grafted with poly(N-isopropylacrylamide). J Biomed Mater Res 27(10): 1243-1251, 1993.

4 Kushida A, Yamato M, Kikuchi A and Okano T: Twodimensional manipulation of differentiated Madin-Darby canine kidney (MDCK) cell sheets: The noninvasive harvest from temperature-responsive culture dishes and transfer to other surfaces. J Biomed Mater Res 54(1): 37-46, 2001.

5 Sekine H, Shimizu T, Dobashi I, Matsuura K, Hagiwara N, Takahashi M, Kobayashi E, Yamato M and Okano T: Cardiac cell sheet transplantation improves damaged heart function via superior cell survival in comparison with dissociated cell injection. Tissue Eng A 17(23-24): 2973-2980, 2011.

6 Akimoto J, Takagi S, Nakayama M, Arauchi A, Yamato M and Okano T: Transplantation of cancerous cell sheets effectively generates tumor-bearing model mice. J Tissue Eng Regen Med 10(10): E510-E517, 2016.

7 Arauchi A, Yang C-H, Cho S, Jarboe EA, Peterson CM, Bae YH, Okano $\mathrm{T}$ and Janát-Amsbury MM: An immunocompetent, orthotopic mouse model of epithelial ovarian cancer utilizing tissue engineered tumor cell sheets. Tissue Eng C 21(1): 23-34, 2014.

8 Johansson L: Histopathologic classification of lung cancer: relevance of cytokeratin and TTF-1 immunophenotyping. Ann Diagn Pathol 8(5): 259-267, 2004.

9 Brown D and Gatter K: Monoclonal antibody Ki-67: its use in histopathology. Histopathology 17(6): 489-503, 1990.

Received November 14, 2017

Revised November 27, 2017

Accepted November 28, 2017 